\title{
EXTENSA FALHA CUTÂNEA E CRANIANA EM PACIENTE COM APLASIA CUTIS CONGENITA
}

\author{
José Gilberto de Brito Henriques¹, Geraldo Pianetti Filho², \\ Alexandre Varella Giannetti ${ }^{3}$, Karina Santos Wandeck Henriques ${ }^{4}$
}

\begin{abstract}
RESUMO - Aplasia cutis congenita (ACC) é doença rara, caracterizada pela ausência de formação completa da pele. Geralmente ocorre no couro cabeludo, na linha mediana, e apresenta-se ao nascimento como uma ferida ulcerada que pode atingir diferentes profundidades e envolver o periósteo, crânio e dura-máter. Descrevemoso caso de menina recem-nascida que apresentava fácies dismórfica com defeito no couro cabeludo que abrangia a quase totalidade da abóbada craniana e apresentava falha óssea desde a base dos ossos frontaisaté osoccipitaise mastóides. A dura-máter estava em sua maior parte exposta, sem sinais de fissuras. Devido à raridade da ACC e ao pequeno número de pacientes nas séries publicadas na literatura a padronização do tratamento ainda é incipiente. O que existem são recomendações. Ainda são necessários estudos que abordem desde a etiologia da doença até a avaliação dos métodos de tratamento e evolução de grupos maiores de pacientes.
\end{abstract}

PALAVRAS-CHAVE: aplasia cutis congenita, tratamento, acrania.

\section{Large scalp and skull defect in patient with aplasia cutis congenita}

\begin{abstract}
Aplasia cutis congenita is a rare condition characterized by the absence of skin layers. It is most common on the scalp, middle line, and it can be seen as a congenital ulcer involving periosteum, skull and dura. We present the case of a female newborn infant with a dysmorphic facies, a large scalp and skull defect exposing the dura. There was no cerebrospinal fluid leakage. The rarity of cases with large defects and small series reported make difficult to determinate the ideal treatment for aplasia cutis congenita. More studies are necessary to define the etiology and best management of this patients.
\end{abstract}

KEY WORDS: aplasia cutis congenita, treatment, acrania.

Aplasia cutis congenita (ACC) é doença rara, caracterizada pela ausência de formação completa da pele. Geralmente ocorre no couro cabeludo, na linha mediana, e apresenta-se ao nascimento como uma ferida ulcerada que pode atingir diferentes profundidadese envolver o periósteo, crânio e dura-máter. A primeira descrição da ACC foi feita por Cordon em 1767 que relatou lesão no membro superior do paciente ${ }^{1}$. Em 1826 Campbell publicou o primeiro relato da lesão em couro cabeludo ${ }^{2}$. Em $75 \%$ dos paciente a ACC ocorre como lesão única ${ }^{3}$. O couro cabeludo é o local de ocorrência em $84 \%$ dos pacientese o crânio é acometido de 15\% a 30\% das vezes ${ }^{3-5}$. Existem diversas teorias sobre a etiopatogenia da ACC, porém nenhuma delas foi devidamente confirmada. A incidência relatada é de
1 a cada 10000 nascimentos ${ }^{6}$. Lesões extensas são ainda mais raras.

É descrito caso de paciente com ACC que acometia o couro cabeludo e a calota craniana em toda a sua extensão.

\section{CASO}

Paciente do sexo feminino, nascida de parto vaginal com 33 semanas e peso adequado para idade gestacional. A mãe tinha 28 anose esta era sua quarta gestação. Houve dois abortos prévios de causa desconhecida e sua filha mais velha, fruto de relação com outro parceiro, era saudável. Durante a gestação a ultra-sonografia evidenciou imagem sugestiva de microcefalia e encefalocele. Ao nascimento (Fig 1) a paciente estava estável, ventilando espontaneamente, movendo os quatro membros. Apresentava facies dismórfica com defeito no couro ca-

${ }^{1}$ Médico Residente de Neurocirurgia do Hospital das Clínicas da Universidade Federal de Minas Gerais, Belo Horizonte MG, Brasil (UFM G); ${ }^{2}$ Professor do Departamento de Neurologia da Faculdade de Medicina da UFMG, Coordenador da Neurocirurgia Pediátrica do Hospital das Clínicas da UFM G; ${ }^{3}$ Preceptor da Residência de Neurocirurgia do Hospital das Clínicas da UFM G; ${ }^{4}$ Médica Residente de Pediatria do Hospital das Clínicas da UFMG.

Recebido 9 Fevereiro 2004, recebido na forma final 4 Maio 2004. Aceito 5 J ulho 2004.

Dr. José Gilberto de Brito Henriques - Avenida Augusto de Lima 196/902 - 30190-001 Belo Horizonte MG - Brasil. E-mail: henriquesjgb@hotmail.com 


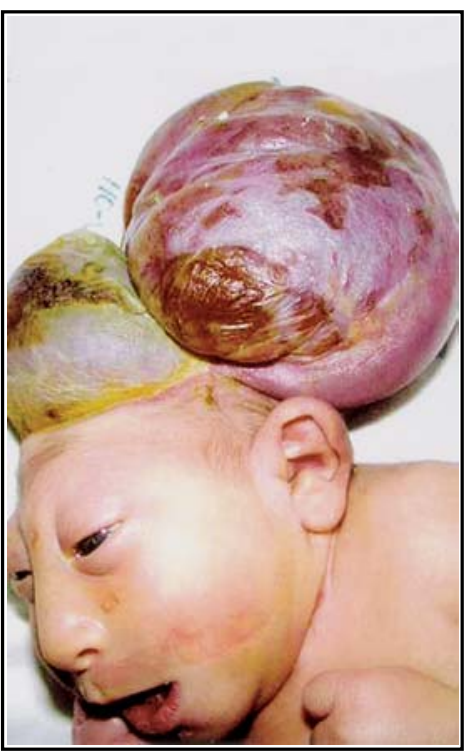

Fig 1. Paciente aos 2 dias de vida. Ausência de pele na abóbada craniana com osso palpado apenas na base do frontal.

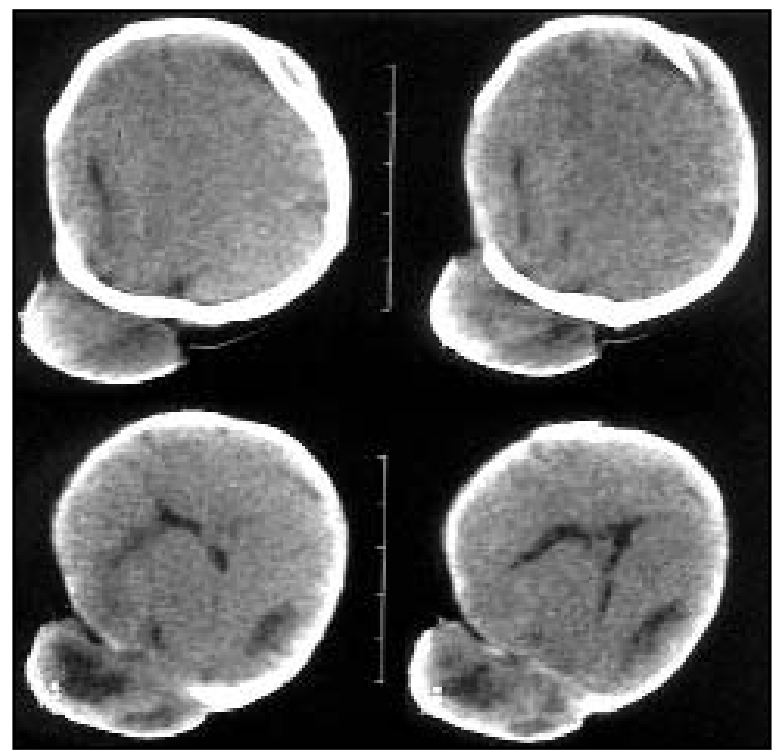

Fig 2. TC de crânio. Tecido apresentando falhas nos ossos temporaise parietais. Encefalocele parieto-occipital, anatomia ventricular distorcida.

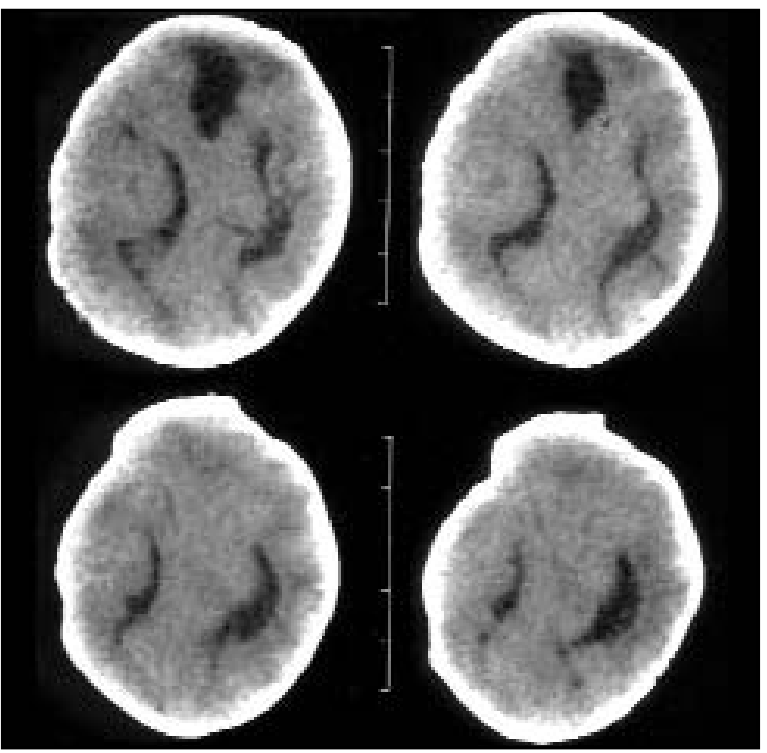

Fig 3. TC de crânio. Falha óssea importante. Esquizencefalia frontal.

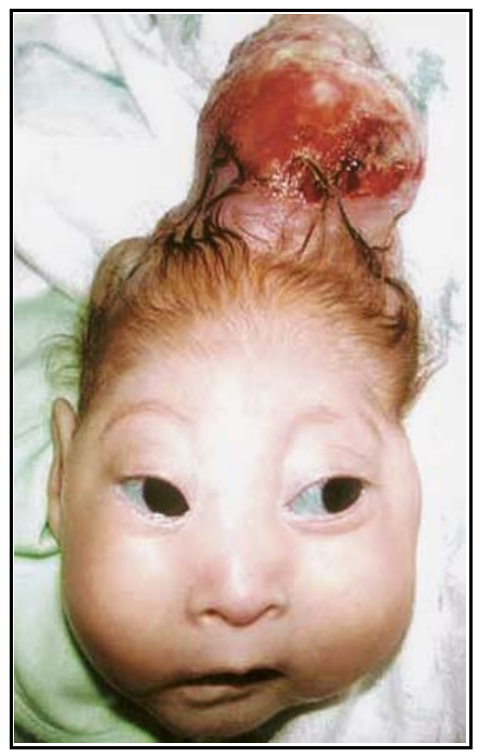

Fig 4. Paciente ao 22 dias de vida. Melhora importante do tecido cutâneo com epitelização do couro cabeludo. beludo que abrangia a quase totalidade da abóbada craniana e apresentava falha óssea desde a base dos ossos frontais, envolvendo a maior pate dos parietais, até os occipitaise mastóides. A dura-máter estava em sua maior parte exposta, sem sinais de fissuras, e havia sítios de tecido de granulação muito friáveis que a recobriam. Devido à ausência da calota craniana o tecido cerebral e a dura-máter se projetavam pela falha óssea causando impressão de uma encefalocele gigante, bilobulada. Na região parietal direita, recoberta por pele integra, havia lesão sugestiva de encefalocele. Não foram percebidos sinais de fístula liquórica apesar de toda a ferida apresentar-se constantemente umidificada.

O exame do cariótipo da paciente foi normal - 46XX. 


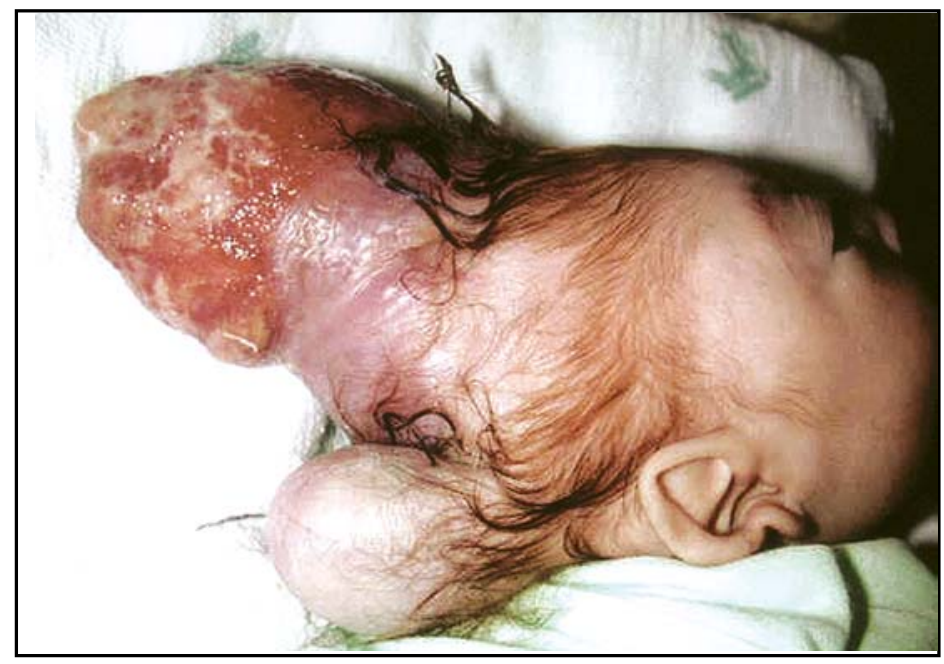

Fig 5. Paciente aos 22 dias de vida. Encefalocele parieto-occipital.

A tomografia computadorizada do crânio (Figs 2e 3) confirmou a falha óssea e a encefalocele parieto-occipital direita. Havia imagem sugestiva de esquizencefalia frontal de lábio aberto e irregularidade do parênquima encefálico devido a aparente ausência da fissura inter-hemisférica. A anatomia ventricular era bastante distorcida. Não havia outras alterações percebidas ao exame físico ou à propedêutica complementar.

Com 20 horas de vida a paciente iniciou quadro febril e queda da freqüência cardíaca. Foi diagnosticada sepse neonatal e iniciada terapia antibiótica. Devido ao quadro séptico e instabilidade da paciente o tratamento conservador foi iniciado: curativosembebidosem solução de doreto de sódio a 0,9\% e sulfadiazina de prata a $1 \%$.

A paciente evoluiu bem com regressão do quadro febril e estabilidade hemodinâmica até o 150 dia de vida quando apresentou ictus tônico-clônico generalizado e episódios de apnéia. Medicação anti-convulsivante foi iniciada com boa resposta. No $22^{\circ}$ dia de vida (Figs 4 e 5), apesar da formação progressiva de tecido de granulação e aparecimento de pele íntegra no couro cabeludo, apresentou secreção purulenta, com odor fétido na ferida. A cultura do material mostrou Klebsiella pneumoniae, Klebsiella oxytoca, Staphylococcus aureus e Corinebacterium sp. Novo ciclo de antibióticos foi iniciado e houve importante melhora da ferida. Houve pequeno sangramento no vértex no $29^{\circ}$ dia de vida que foi controlado com compressão local. O quadro geral da paciente mantendo-se satisfatório, com boa evolução até o 67ำ dia de vida, quando teve episódio de vômito seguido de cianose e bradicardia que foi refratária ao tratamento instituído com evolução para o óbito.

\section{DISCUSSÃO}

Diversasteoriastentam explicar a etiopatogenia da ACC: defeitos no fechamento do tubo neural; necrose por pressão localizada na pele do embrião e mesênquima adjacente; śfiliscongênita (que foi descartada); adesões amnióticas; anomalias vasculares ${ }^{5-9}$. As heranças autossômicas dominante e recessiva também já foram descritas 4,6,7,9,10. A concomitância da ACC com outras malformações originou alguns sistemas de classificação baseadosna morfologia da lesão, na localização e nas outras malformações presentes ${ }^{11-13}$. Segundo Glasson e Dun$\mathrm{can}^{7}$, a ACC geralmente ocorre no primeiro filho do casal e no sexo feminino. A maioria das lesões da ACC é única ${ }^{3}$, acomete somente a epiderme ${ }^{7,9,10}$, tem forma oval ou estrelada com menos de dois centímetrose ocorre principalmente no vértex, seguido de outros locais no couro cabeludo, antebraços, joelhos, tronco e pescoço ${ }^{5}$.

A histologia das lesões superficiais mostra ausência da epiderme, de seusanexose atrofia da derme. Aslesões profundasà microscopia mostram tecido de granulação e cicatricial em diferentes fases evolutivas.

As lesões extensas e as que acometem a duramáter são extremamente rarase têm mortalidade elevada, sendo as infecçõese hemorragiasas principais causas de morte nestes pacientes, com taxa de mortalidade de $20 \%$ e $55 \%$ respectivamente ${ }^{3,6,10}$. As outras causas de morte descritas são trombose de seio venoso, hiponatremia (por perda direta de sódio pela ferida), hipercalemia (devido ao uso de sulfadiazina de prata nos curativos) ${ }^{6,7}$. Desta maneira, a mortalidade é dependente da extensão e da profundidade das lesões, além dos casos de associação com outras malformações. A concomitância com encefalocele, mielomeningocele e outros disrafismostambém pode ocorrer ${ }^{8}$. Ribuffo et 
al. publicaram o relato de caso de um paciente que, com o tratamento conservador, desenvolveu por duasvezesencefalocele no local da $\mathrm{ACC}^{10}$. Higginbottom et al. descreveram cinco pacientes com ACC em região lombo-sacra, destes; quatro apresentavam medula presa à exploração cirúrgica ${ }^{14}$. O diagnóstico diferencial da ACC deve ser feito com os disrafismos crânio-espinhais, abscessos do couro cabeludo, tocotraumatismose tecido cerebral heterotópico 5,9 .

A história natural da ACC é de cicatrização de lesões pequenas e hemorragiase infecções nas lesões grandes. Devido à evolução favorável das lesões pequenase sem acometimento da dura-máter o tratamento recomendado é o conservador, a base de curativose cuidados com a lesão. O maior desafio terapêutico encontra-se naslesões de grande extensão e com envolvimento da dura-máter. Alguns autores advogam o tratamento conservador mesmo nas lesões extensas devido à grande dificuldade técnica e aos relatos de boa evolução de alguns pacientes ${ }^{5}$. Outros autores ${ }^{3,5}$ sugerem o tratamento conservador inicialmente com cirurgia precoce no caso de pequenos sangramentos pois é bem difundida a idéia de que os pequenos sangramentos precedem grandes hemorragias ${ }^{3,6,7}$. Estes sangramentos, ocorrem devido a pequenas fissuras secundárias ao ressecamento da superfície das lesões após os curativos. Cirurgias de urgência devido a lesões do seio sagital superior ou de lagos venosos são extremamente difíceis, dadas as condições do tecido a ser suturado e a anatomia anômala das estruturas vasculares ${ }^{7}$.

É consenso na literatura que as lesões extensas devem ser tratadas cirurgicamente. As grandes dificuldades técnicas para o tratamento cirúrgico são: a escassez de tecido cutâneo íntegro para planejamento de retalho; a má adaptação dos retalhos cutâneos sobre o leito da ferida devido à vascularização anômala e ao tecido de granulação já formado sobre a ferida ${ }^{6}$; a absorção dos enxertos ósseos que pode ocorrer quando estes são colocados sobre enxertos de dura-máter. Além destas, os diversos procedimentos cirúrgicos de grande porte nas crianças tornam a decisão pela cirurgia ainda mais difícil. No entanto há relatos de bons resultados de reconstituição do couro cabeludo com retalhosautólogos e expansores de tecidos ${ }^{3-6}$. Ascranioplastias autólogas ou heterólogas foram realizadas em abordagens precoces e mais tardias devido ao pouco crescimento dos ossos do crânio após a cicatrização da pele ${ }^{5}$.

A opção pelo tratamento conservador da paciente aqui relatada foi feita devido à pouca quantidade de tecido íntegro para o retalho cutâneo e ao quadro séptico precoce.

Devido à raridade da $\mathrm{ACC}$ e ao pequeno número de pacientes nas séries publicadas na literatura, a padronização do tratamento ainda é muito inicial. O que existem são recomendações. Ainda são necessários estudos que abordem desde a etiologia da doença até a avaliação dos métodos de tratamento e evolução de gruposmaiores de pacientes.

\section{REFERÊNCIAS}

1. Cordon M. Extrait d'une lettre au sujet de trois enfants de la même mère nés avec partie des extrémités dénuée de peau. J Méd Chir Pharmacie 1767;26:556-557.

2. Campbell W. Case of congenital ulcer on the cranium of a fetus, terminating in fatal hemorrhage, on the $18^{\text {th }}$ day after birth. Edinb J Med Sci 1826;2:82-83.

3. Moscona R, Berger J, Govrin J. Large skull defect in aplasia cutis congenita treated by pericranial flap: long term follow-up. Ann Plast Surg 1991;26:178-182.

4. Argenta LC, Dingman RO. Total reconstruction of aplasia cutis congenita involving scalp, skull and dura. Plast Reconstr Surg 1986;77:650-653.

5. Yang JY, Yang WG. Large scalp and skull defects in aplasia cutis congenita.Br J Plast Surg 2000;53:619-622.

6. Bajpai M, Pal K. Aplasia cutis cerebri with partial acrania: total reconstruction in a severe case and review of literature. J Ped Surg 2003;38:1-3.

7. Glasson DW, Duncan GM. Aplasia cutis congenita of the scalp: delayed closure complicated by massive hemorrhage. Plast Reconstr Surg 1985;75:423-425.

8. Six EG, Kelly DL. Conservative management of aplasia cutis congenita: case report. Neurosurgery 1981;8:233-235.

9. Stephan MJ, Smith DW, Ponzi JW, Alden ER. Origin of scal vertex aplasia cutis. J Pediat 1982;101:850-853.

10. Ribuffo D, Constantini M, Gullo P, Houseman ND, Taylor GI. Aplasia cutis congenita of the scalp, the skull and dura. Scand J Plast Reconst Surg Hand Surg 2003;37:176-180.

11. Frieden IJ. Aplasia cutis congenita: a clinical review and proposal for classification. J Am Acad Dermatol 1986;14:646-660.

12. Evers ME, Steijlen PM, Hamel BC. Aplasia cutis congenita and associated disorders: an update. Clin Genet 1995;47:295-301.

13. Kosnik EJ, Sayers MP. Congenital scalp defects: aplasia cutis congenita J Neurosurg 1975;42:32-36.

14. Higginbottom MC, Jones KL, James HE. Aplasia cutis congenita: a cutaneous marker of occult spinal dysraphism. J Pediat 1980;96:687-689. 